\title{
Endoscopic Ultrasound-guided Radio-frequency Ablation for Pancreatic Cancer
}

\author{
Pradermchai Kongkam, Rungsun Rerknimitr \\ Gastrointestinal Endoscopy Excellence Center and Division of Gastroenterology, Department of Medicine, Faculty of Medicine, Chulalongkorn \\ University and King Chulalongkorn Memorial Hospital, Thai Red Cross Society, Bangkok, Thailand
}

Prognosis of unresectable pancreatic cancer is poor with the rate of surviving more than 5 years is less than $10 \%$ despite multi-modalities treatment. The American Society of Clinical Oncology suggested that all patients with metastatic pancreatic cancer should be offered information about clinical trials. Endoscopic ultrasound guided radio frequency ablation has been recently used in patients with advanced stage pancreatic cancer in a few studies. This article has reviewed information from published articles using endoscopic ultrasound guided radio frequency ablation for advanced pancreatic cancer.

Korean J Pancreas Biliary Tract 2021;26(2):98-104

Keywords: Pancreatic neoplasms, Endoscopic ultrasound guided radio frequency ablation $\begin{array}{lll}\text { Received } & \text { Jan. 21, } 2021 \\ \text { Revised } & \text { Feb. 15, } 2021 \\ \text { Accepted } & \text { Feb. 22, } 2021\end{array}$

Corresponding author : Rungsun Rerknimitr Gastrointestinal Endoscopy Excellence Center and Pancreas Research Unit, Department of Medicine, Faculty of Medicine, Chulalongkorn University and King Chulalongkorn Memorial Hospital, Thai Red Cross Society, 1873 Rama 4 Road, Pathumwan, Bangkok 10330, Thailand

Tel. +66-2-256-4356 Fax. +66-2-652-4219 E-mail; ercp@live.com ORCID: https://orcid.org/0000-0001-6866-6886

\section{INTRODUCTION}

Although pancreatic cancer is not commonly found as the cause of gastrointestinal symptoms, those who presented with symptoms have already reached a critical stage, making it is impossible to be cured with surgery in majority of the patients. Therefore, the prognosis of the disease is poor; the rate of surviving more than 5 years is less than $10 \%$. ${ }^{1}$ Statistically evidence indicates that pancreatic cancer is the eighth and ninth leading cause of death among male and female patients worldwide respectively. ${ }^{2}$ The only curative treatment for pancreatic cancer is surgery, but this is not suitable for those with advanced stage even early locoregional advancement cases. Traditionally, patients would be referred to chemotherapy and/ or radiotherapy. Such practices are aimed at extending the length survival of patients and improving their quality of life at best. $^{3}$

The use of radio frequency wave to ablate stage III pancreatic cancer has been used by surgeons who perform laparotomy to treat patients with pancreatic cancer, where it was not possible for the disease to be cured with surgery. ${ }^{4}$ However, such procedure is considered as cumbersome because laparotomy is still required and not cost effective, it is hence not widely adopted. 
During the past 5 years, needle electrodes have been improved to feature as a longer cable and can be connected to the radio frequency generator. This enables them to be inserted through the gastrointestinal echoendoscope to directly reach the pancreas. Because of these advantages, researchers started adopting endoscopic-ultrasound guided radio-frequency ablation (EUS-RFA). ${ }^{5}$ However, as the system is still not widely available, data regarding pancreatic cancer treatment is therefore limited. The authors of this paper are interested in EUS-RFA for pancreatic cancer and have developed their experience with this new invention. In this review, information obtained from previous studies will also be discussed, along with the experience of the authors.

\section{MAIN BODY}

\section{Tools}

Presently, a radiofrequency generator and a needle electrode inserted via echoendoscope are manufactured by four companies. ${ }^{6}$ However, the model which is the most popular for EUS-RFA of pancreatic lesions, especially for cancer, is the one manufactured in Korea (STARmed, Goyang, Korea) (19-gauge needle: $1.1 \mathrm{~mm}$ ). The generator is a box with a channel for the needle electrode to be inserted through. Radiofrequency wave will be transmitted via the needle into the pancreatic lesion.
The needle has been designed in a manner similar to a biopsy needle, but without a hole at the tip to probe into body tissue. It has a solid and pointed tip, allowing endosonographers to insert this $150-\mathrm{cm}$-long device into the channel of the echoendoscope. Endosonographers would utilize images obtained from sound waves to navigate the needle into pancreatic lesions. The tip of the needle, $1 \mathrm{~cm}$ in length, is not insulated, which allows direct emission of the radio frequency waves into the area for ablation. Radio frequency emits heat which can damage tissues and eventually ablate them. The generator is equipped with an internal cooling system (Fig. 1).

\section{Operation procedures at the King Chulalong- korn Memorial Hospital (KCMH)}

1) The endosongrapher inserts the needle electrode via the echoendoscope into the patient's abdomen. Sonographic waves will then be utilized to determine the location of the lesion requiring ablation in the pancreas. During this time, the endosonographer will also examine whether there are any interposing blood vessels along the path on which the needle will be inserted. If there is none, the endosonographer will proceed with the next step. Another practical point is that, if endosonographer anticipate that the ablated area will be within $5 \mathrm{~mm}$ in distance from the main pancreatic duct, a prophylactic pancreatic stent insertion is recommended.
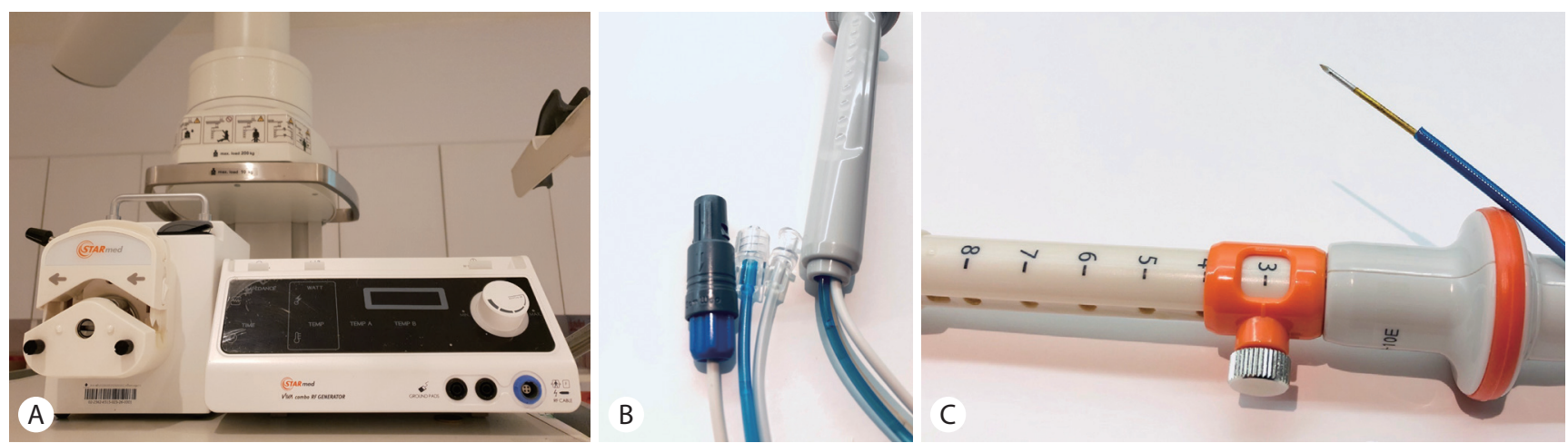

Fig. 1. (A) Displays a radio frequency generator (on the right side). The white cable serves as the transmission line. The device on the left is a viva pump that carries water through the blue cable into the needle electrode for cooling, while the endosonographer is performing tumor ablation. (B) Illustrates the extension of the needle, with a white cable from the radio frequency generator and a blue cable which carries water for cooling. The white cable is connected to the patient's body to complete the transmission cycle. (C) Displays the handle of the needle electrode, with a round button to select and secure the needle length. 
2) The medical assistant turns on the needle electrode and installs the built-in cooling system to the radio frequency generator and turns on the radio frequency generator. At $\mathrm{KCMH}$, the authors and the team use the continuous mode, setting the maximum resistance at 50 watts.

3) The endosonographer inserts the needle electrode through the echoendoscope and attaches it into the lesion similar to the standard technique of EUS-guided needle biopsy. When the needle reaches the desired position, the endosonographer then controls the emissions and pauses of the radio frequency wave. Practically, transgastric ablation of tumors is easier than transduodenal approach due to limited flexibility of the EUS-RFA needle. Fortunately, given most recruited pancreatic cancers are in an advanced stage, transgastric EUS-RFA is hence possible and preferred. Nevertheless, no study has reported the difficulty of transduodenal EUS-RFA. In most cases, endosonographers would determine the size of the tumor using sonographic imaging. The tumor will feature a more hypoechoic shade than its surrounding tissues. Perhaps, additional technologies may be employed as well, for instance, elastography, which is used to measure the level of hardness of the target lesion. It is well known that pancreatic cancer cells are solid. Therefore, by using elastography, the cancer area will appear in blue, specifying more solid lesions. The softer ones are displayed in green and red, respectively (Fig. 2).

4) At $\mathrm{KCMH}$, the endosonographer will ablate the lesion until it appears hyperechogenic representing post heated tumor. The endosonographer who perform the procedure will verify that the white image (Fig. 2) covers the desired area as much as possible and is at least $5 \mathrm{~mm}$ away from the bile ducts, pancreas and blood vessels to avoid injury to these structures. In the King Chulalongkorn Memorial Hospital, we used continuous mode, setting maximum power at 50 watts through the 19-gauge needle type EUS-RFA probe.

5) After the desired area has been completely ablated (until an echogenic image is seen), the endosonographer will position the needle electrode in the next area and procedures will be repeated until little to no unablated area is left. The endosonographer then removes the needle and examines the lesion using the echoendoscope. If unablated area (original heterogeneous hypoechoic area) can still be observed, the endosonographer will repeat the methods until no more lesions can be seen or until the procedure cannot be continued due to interposing blood vessels or ducts or post ablative hyperechogenic area obscuring the target area. Procedural time is not limited as long as all vital signs are stable enough for the procedure. Area in farer side will be firstly ablated followed by the nearer area.

6) In 2-4 weeks, the endosonographer makes an appointment for the patient to repeat EUS-RFA. If unablated lesions are still detected, it will be ablated again with another needle electrode. This process will be repeated until the whole lesion is completely ablated. To our observation, post ablated areas will become either hyperechoic areas in early days or anechogenic areas due to necrosis of the tumor in later days. In $\mathrm{KCMH}$, the authors and the team perform the follow-up examination with computed tomography. If more lesion is detected, additional
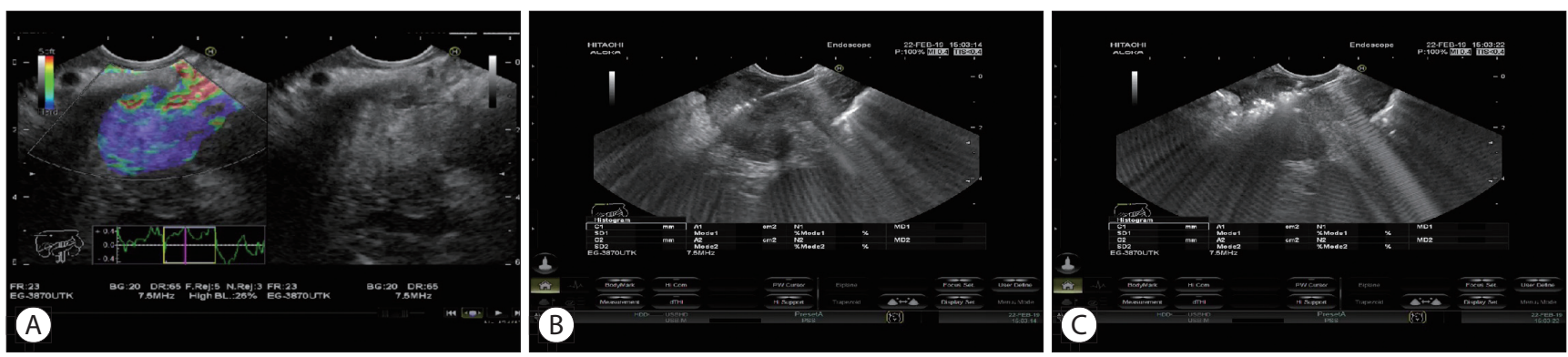

Fig. 2. (A) Illustrates pancreatic cancer lesions examined using elastography to identify the solidness of cancer cells, in order to determine the size of tumor before ablating the lesions with a needle electrode. It can be noticed that with elastography, areas with more solid lesions will be shown in blue, allowing the endosonographer to determine the size of lesions. (B) Displays a photo of the needle electrode, which is an echogenic straight line, inserted into the hypoechoic cancer lesions. (C) Illustrates the endosonographer emitting radio frequency and heating the lesions. After being heated, the lesion area will be seen as a hyperechogenic area, expanding from the tip of the needle. 
ablation will be carried out 2-3 months after the beginning period of lesion assessment with the oncology team to decide whether the treatment will be continued alongside with other methods, such as chemo-radiotherapy.

\section{Efficacy of EUS-RFA for pancreatic cancer (Table 1)}

There have been only a handful of studies reported as cases series examining the efficacy of EUS-RFA for pancreatic cancer are illustrated as follows. Currently, all studies focus on feasibility of EUS-RFA procedures and tumor necrosis as main clinical outcomes. No study thus far evaluated the effect of EUS-RFA of pancreatic cancer for survival time, downstaging effect, etc.

A cases series by Song et al. ${ }^{5}$, published in 2016, examined four patients with pancreatic cancer and two patients with metastatic cancer to the pancreas. The average tumor size was $38 \mathrm{~mm}(30-90 \mathrm{~mm})$. The procedure was achieved using a 20-gauge needle and device from STARmed, manufactured in Korea. The power was set at 20-50 watts and application time was 10 seconds. The average ablation time was 1.3 times. Expected result was necrosis at the ablated area. All target lesions were successfully ablated with necrosis in all of them. This study reported complications in two patients with mild back pain after the procedure. ${ }^{5}$

The second study, published in 2018, concerns EUS-RFA for pancreatic cancer in nine patients; eight of which had pancreatic cancer and the other had metastatic kidney cancer spread to the pancreas. The procedure featured an 18-gauge needle also from STARmed, manufactured in Korea. This study had to exclude one patient as a large necrotic area containing fluid portions inside the tumor was detected during EUS. The endosonographer was able to complete the procedure in the remaining eight patients with ablated areas inside tumors in all patients. There were three patients with mild abdominal pain after the procedure. In the remaining patients, no complications occurred at all, even after the average 6-month followup. ${ }^{7}$

The third study, published in 2018, recruited 10 patients with unresectable non-metastatic pancreatic cancer for EUS-RFA (STARmed) (18-gauge needle) following neoadjuvant chemotherapy. No major adverse events. Necrosis inside target lesions were seen in all cases. The authors concluded that EUS-RFA is feasible and minimally invasive. ${ }^{8}$

A recent systematic review of 38 patients with unresectable pancreatic cancer reported technical and clinical success rate at $100 \%(38 / 38)$ and $(26 / 26))^{9}$ The technical success was defined as a successful placement of the EUS-RFA probe into the target lesions, and the clinical success was defined as necrosis of target lesion by EUS-RFA. In this systematic review, which recruited several kinds of pancreatic tumor including pancreatic adenocarcinoma $(\mathrm{n}=38)$ pancreatic neuroendocrine neoplasm $(n=53)$, metastasis to the pancreas $(n=4)$ and other pancreatic lesions $(n=41)$, reported adverse events rate associated with EUS-RFA at $14.7 \%$, of which, the abdominal pain was the

Table 1. Technical and clinical success rate of endoscopic ultrasound guided radio frequency ablation of pancreatic cancer as well as adverse events rate

\begin{tabular}{|c|c|c|c|c|c|}
\hline Study & Study type & Number of patients & Technical success $(n)$ & Clinical success ( $\mathrm{n}$ ) & Adverse events (n) \\
\hline Song et al..$^{5}$ (2016) & Prospective & $\begin{array}{c}6(\operatorname{PDAC}[n=4], \\
\text { metastasis }[n=2])\end{array}$ & $100 \%(6 / 6)$ & $100 \%(6 / 6)$ & $33.3 \%(2 / 6) ;$ mild abdominal pain \\
\hline Crinò et al.' (2018) & Prospective & $\begin{array}{l}8(\operatorname{PDAC}[\mathrm{n}=7] \\
\text { metastasis }[\mathrm{n}=1])\end{array}$ & $100 \%(8 / 8)$ & $100 \%(8 / 8)$ & $37.5 \%$ (3/8); mild abdominal pain \\
\hline Scopelliti et al. ${ }^{8}$ (2018) & Prospective & $10(\operatorname{PDAC}[\mathrm{n}=10])$ & $100 \%(10 / 10)$ & $100 \%(10 / 10)$ & $\begin{array}{l}\text { 40\% (4/10); asymptomatic ascites }(n=2) \text {, } \\
\text { asymptomatic peripancreatic effusion } \\
(n=2)\end{array}$ \\
\hline Total & - & $\begin{array}{l}24(\operatorname{PDAC}[n=21] \\
\text { metastasis }[n=3])\end{array}$ & $100 \%$ & $100 \%$ & $37.5 \%(9 / 24)$; alls were mild events \\
\hline
\end{tabular}

PDAC, pancreatic ductal adenocarcinoma 
most common event at $9.8 \%{ }^{9}$ Based on our institutional experience and results from prior studies, neither death nor serious adverse events occurred from the result of the EUS-RFA procedure. In our experience, the only one adverse event that we had was mild pancreatitis which was successfully treated with conservative treatment.

\section{Relieving abdominal pain from pancreatic cancer}

One condition that these patients with terminal pancreatic cancer do suffer is abdominal pain and many of them ended up with narcotic addiction due to severe and uncontrolled pain. Another method of relieving abdominal pain due to pancreatic cancer is ablating the celiac nerve by alcohol to reduce the pain. This can be achieved through two methods. The first is a percutaneous approach carried out by an anesthesiologist. Another method is done via endoscope by an endoscopist. The endosonographer will use the echoendoscope to locate the origin of the celiac artery, then injects substance near the origin of the artery to damage its nerves. The overall efficacy of this procedure is $45-70 \%{ }^{10-13}$ Presently, there is no comparative study regarding the efficacy between percutaneous and endoscopic approaches. Study by Bang et al. ${ }^{14}$ in 2018 featured a single-blind, randomized trial to compare the efficacy of EUS-guided ethanol ablation and EUS-RFA in ablating celiac nerve. The authors performed both procedures in patients with pancreatic cancer who were not eligible for surgery or in terminal stage. Fourteen patients underwent EUS-guided ethanol ablation and twelve underwent nerve ablation with EUS-RFA. The needle electrode used by Bang et al. ${ }^{14}$ was very small with a size of 1 French 1 Fr (0.33 m, 0.013”) (HabibTM EUS-RFA catheter: EMcision, London, UK). The researcher utilized a 19-gauge biopsy needle to penetrate the celiac ganglion nerve and inserted the needle electrode into the nervous system. The primary variables evaluated by the researchers were the intensity of pain at 2 and 4 weeks after treatment, using standard of the European Organization for

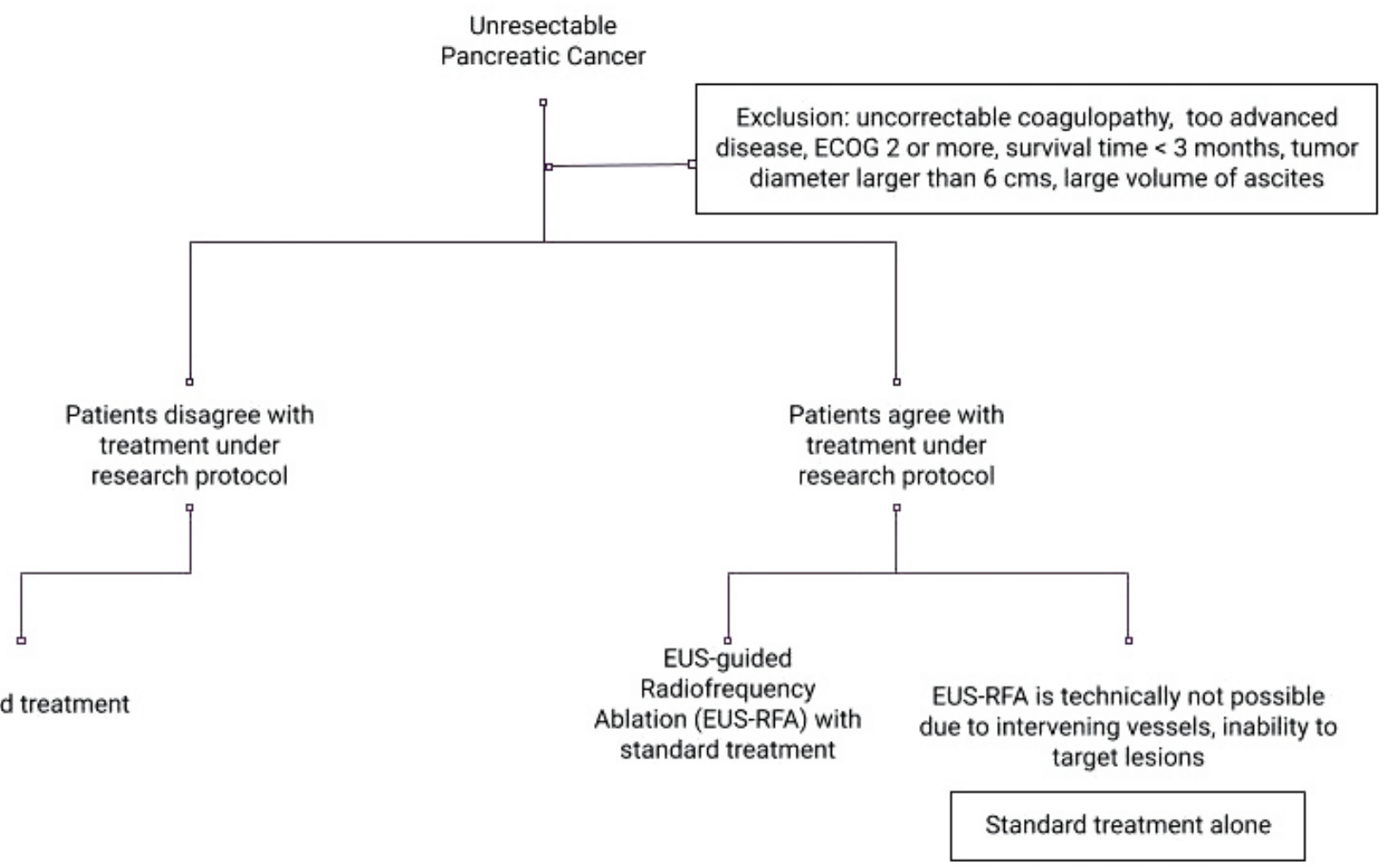

Fig. 3. The role of endoscopic ultrasound-guided radiofrequency ablation of pancreatic cancer based on evidence from this review. 
Research and Treatment of Cancer and the quality of life questionnaire of pancreatic cancer module (PAN26). Results from the study showed that patients who underwent treatment with EUS-RFA experienced significantly less pain than those treated with ethanol ablation $(51.9 \%$ versus $64.4 \%$, respectively, $p=0.032) .{ }^{14}$ Given the current most popular EUS-RFA probes is a 19-gauge EUS-RFA needle which is too large to ablate only celiac nerve, it is interesting to see effect of EUS-RFA for pancreatic cancer in terms of pain reduction as a 19-gauge probe might incidentally cover area of celiac nerve during procedure.

\section{Indications and contraindications}

Based on our experience, current indications and contraindications are as follows.

Indications

- Unresectable pancreatic cancer

- Eastern Cooperative Oncology Group score 0-2

- Expected survival time longer than 3 months

- Aim for palliative treatment

Contraindications

- Significant ascites

- Pregnancy

- Uncorrectable coagulopathy

- Intervening vessels

\section{CONCLUSIONS}

From aforementioned data, a few studies have proven that the EUS-RFA for pancreatic cancer is easy to use with the high successful rate in ablation induced tumor necrosis, and also pain reduction. So far, the method is not yet proven in terms of increasing the survival rate or extending the life of patients. We have created an algorithm of EUS-RFA in clinical practice based on current evidence as shown in Fig. 3. As of now, it is still too early to advise patients to undergo EUS-RFA as a standard procedure. Physicians may suggest that if patients are interested in receiving this treatment, they should participate in its research phase in order to consider the pros and cons, especially with randomized and anonymous research, and to evaluate the results of this treatment as such information will still be much needed in the near future.

\section{Conflicts of Interest}

The authors have no conflicts to disclose.

\section{Acknowledgments}

This work was supported by the Pancreas Research Unit, Faculty of Medicine, Chulalongkorn University.

\section{REFERENCES}

1. Kongkam $P$, Benjasupattananun $P$, Taytawat $P$, et al. Pancreatic cancer in an Asian population. Endosc Ultrasound 2015;4:56-62.

2. Siegel R, Ward E, Brawley O, Jemal A. Cancer statistics, 2011: the impact of eliminating socioeconomic and racial disparities on premature cancer deaths. CA Cancer J Clin 2011;61:212-236.

3. Sohal DPS, Kennedy EB, Khorana A, et al. Metastatic pancreatic cancer: ASCO clinical practice guideline update. J Clin Oncol 2018;36:2545-2556.

4. Girelli R, Frigerio I, Giardino A, et al. Results of 100 pancreatic radiofrequency ablations in the context of a multimodal strategy for stage III ductal adenocarcinoma. Langenbecks Arch Surg 2013;398:63-69.

5. Song TJ, Seo DW, Lakhtakia S, et al. Initial experience of EUS-guided radiofrequency ablation of unresectable pancreatic cancer. Gastrointest Endosc 2016;83:440-443.

6. Lakhtakia S, Seo DW. Endoscopic ultrasonography-guided tumor ablation. Dig Endosc 2017;29:486-494.

7. Crinò SF, D'Onofrio M, Bernardoni L, et al. EUS-guided radiofrequency ablation (EUS-RFA) of solid pancreatic neoplasm using an 18-gauge needle electrode: feasibility, safety, and technical success. J Gastrointestin Liver Dis 2018;27:67-72.

8. Scopelliti F, Pea A, Conigliaro R, et al. Technique, safety, and feasibility of EUS-guided radiofrequency ablation in unresectable pancreatic cancer. Surg Endosc 2018;32:4022-4028.

9. Dhaliwal A, Kolli S, Dhindsa BS, et al. Efficacy of EUS-RFA in pancreatic tumors: is it ready for prime time? A systematic review and metaanalysis. Endosc Int Open 2020;8:E1243-E1251.

10. LeBlanc JK, Al-Haddad M, McHenry L, et al. A prospective, randomized study of EUS-guided celiac plexus neurolysis for pancreatic cancer: one injection or two? Gastrointest Endosc 2011;74:1300-1307.

11. Wyse JM, Battat R, Sun $S$, et al. Practice guidelines for endoscopic ultrasound-guided celiac plexus neurolysis. Endosc Ultrasound 
2017:6:369-375.

12. Sahai AV, Lemelin V, Lam E, Paquin SC. Central vs. bilateral endoscopic ultrasound-guided celiac plexus block or neurolysis: a comparative study of short-term effectiveness. Am J Gastroenterol 2009;104:326329.

13. Gress F, Schmitt C, Sherman S, Ikenberry S, Lehman G. A prospective randomized comparison of endoscopic ultrasound- and computed tomography-guided celiac plexus block for managing chronic pancreatitis pain. Am J Gastroenterol 1999;94:900-905.

14. Bang JY, Sutton B, Hawes RH, Varadarajulu S. EUS-guided celiac ganglion radiofrequency ablation versus celiac plexus neurolysis for palliation of pain in pancreatic cancer: a randomized controlled trial (with videos). Gastrointest Endosc 2019;89:58-66.e3. 